ISSN 0103-5150

Fisioter. Mov., Curitiba, v. 26, n. 1, p. 37-45, jan./mar. 2013

Licenciado sob uma Licença Creative Commons

\title{
Novo dispositivo EPAP subaquático no pós-operatório de cirurgia de revascularização do miocárdio
}

\author{
A novel underwater EPAP device for post- \\ myocardial revascularization surgery
}

\section{Francine Cavalli ${ }^{[a]}$, Percy Nohama ${ }^{[b]}$}

[a] Fisioterapeuta especialista em Fisioterapia Cardiorrespiratória, mestre em Tecnologia em Saúde pela Pontifícia Universidade Católica do Paraná (PUCPR), Curitiba, PR - Brasil, e-mail: francinecavalli@yahoo.com.br

[b] Doutor em Engenharia Elétrica pela Universidade Estadual de Campinas (Unicamp), docente do Programa de PósGraduação em Tecnologia em Saúde da Pontifícia Universidade Católica do Paraná (PUCPR) e do Programa de PósGraduação em Engenharia Elétrica e Informática Industrial da Universidade Tecnológica Federal do Paraná (UTFPR), Curitiba, PR - Brasil, e-mail: percy.nohama@gmail.com

\section{Resumo}

Introdução: A prevalência de cirurgias cardíacas é alta e acarreta custos elevados em decorrência de complicações secundárias. Dentre as principais complicações, estão as alterações pulmonares. A fisioterapia respiratória desempenha função relevante nas intervenções cirúrgicas, atuando na remoção de secreções pulmonares e na reexpansão pulmonar, por meio de tratamento com pressão positiva expiratória final (EPAP). Objetivo: Descrever um novo dispositivo EPAP (pressão positiva expiratória final) subaquático, desenvolvido com o objetivo de reverter atelectasias e complicações pós-operatórias, em pacientes submetidos à cirurgia de revascularização do miocárdio (RM). Materiais e métodos: O dispositivo foi testado em um conjunto amostral reduzido, não probabilístico e intencional, envolvendo 17 pacientes (seis atenderam aos critérios de inclusão), internados na Unidade Coronariana, com idade entre 55 e 65 anos; pós-desmame de ventilação mecânica pós-cirúrgica; lúcidos, orientados e contactuantes; com o sistema cognitivo preservado e sem deformidades orofaciais. Os parâmetros medidos foram: saturação de oxigênio $\left(\mathrm{SpO}_{2}\right)$ e frequência cardíaca, volume expiratório forçado no primeiro segundo $\left(\mathrm{VEF}_{1}\right)$, capacidade vital forçada (CVF), fluxo expiratório máximo, ou Peak Flow (PEF), pressão arterial diastólica e sistólica. Realizou-se, ainda, o exame radiográfico torácico, a ausculta pulmonar e, por observação do fole torácico, a frequência respiratória; ainda, observou-se a queixa principal e o volume de expectoração. Resultados: Os dados espirométricos não mostraram valores significativos, mas 
os sinais vitais mantiveram-se normais. Conclusão: Os resultados obtidos indicam que o EPAP subaquático melhora a saturação de oxigênio, auxilia na remoção de secreções por meio da ventilação colateral e expectoração, otimiza a ausculta pulmonar, reduz a queixa principal "dor" pelo aumento progressivo da mobilidade torácica, tornando a respiração menos superficial. Além disso, reverte atelectasias, tornando-se essencial no tratamento de alterações respiratórias pós-operatórias de revascularização de miocárdio.

Palavras-chave: EPAP subaquático. Alterações respiratórias pós-cirúrgicas.

Revascularização do miocárdio.

\section{Abstract}

Introduction: The prevalence of cardiac surgery is high and results in high costs due to secondary complications. The main complications are pulmonary changes. Respiratory therapy plays a relevant role in surgical interventions, working in the removal of pulmonary secretions and lung reexpansion, by treating with positive end expiratory pressure (PEEP). Objetives: To describe a new underwater PEEP device, in order to revert atelectasis and post-surgery complications in patients submitted to myocardium revascularization surgery (MR). Materials and methods: The device was tested on a small sample set, non-probabilistic and deliberated, composed by 17 patients, hospitalized in the coronary unit of the Cardiology Institute of Santa Catarina, in São José, Brazil, aged between 55-65 years old, post wean of post-surgery mechanical ventilation, conscious, orientated and in contact, with cognitive system preserved and without oral-facial physical deformities. The following parameters were measured: oxygen saturation $\left(\mathrm{SPO}_{2}\right)$ and heart rate, forced expiratory volume in the first second $\left(E V F_{1}\right)$, forced vital capacity (FVC), maximal expiratory flow (MEF) or peak flow (PEF), diastolic and systolic blood pressure. Also, it was performed pulmonary auscultation and measured respiratory frequency. It was also assessed thoracic radiographic images, main complain and volume of expectoration. Results: Spirometric data did not show statistical significance but the vital signs remained normal. Conclusion: underwater PEEP improves oxygen saturation and removal of secretions through collateral ventilation and expectoration; optimizes pulmonary auscultation; reduces the main complaint, "pain", increasing progressively thoracic mobility. Besides, it reverts atelectasis, becoming essential in the treatment of respiratory post-operative MR alterations.

Keywords: Underwater EPAP. Breathing post-surgical changes. Myocardial revascularization.

\section{Introdução}

Uma complicação pós-operatória é definida como uma segunda doença inesperada que ocorre até 30 dias após uma cirurgia, alterando o quadro clínico do paciente que necessita, consequentemente, de intervenção terapêutica medicamentosa ou não (1).

Atualmente, as cardiopatias constituem-se numa das principais causas de morte nos Estados Unidos, com cerca de um milhão de óbitos a cada ano, totalizando $51 \%$ de todas as mortes, e são responsáveis por grande parte das complicações pós-operatórias (2). A prevalência de cirurgia cardíaca é alta no mundo ocidental: cerca de $10 \%$ da população anualmente, acarretando custos elevados pelas complicações secundárias decorrentes das cirurgias cardíacas (3).

As complicações pulmonares constituem a segunda causa mais frequente de morbidade e mortalidade no período pós-operatório de cirurgia cardíaca. As alterações pulmonares surgem a partir de inúmeros fatores, principalmente em virtude do uso de circulação extracorpórea, que ocasiona discretas alterações metabólicas e eletrolíticas no paciente, deixando-o mais suscetível a infecções (4). Medicações anestésicas ou pré-anestésicas que incluem os depressores do sistema nervoso central e secantes de mucosa comprometem o clearence pulmonar e os reflexos respiratórios mediados pelo centro bulbar. Além disso, o período pós-operatório é caracterizado por dor, imobilidade no leito, restrição da respiração profunda e diminuição do estímulo da tosse. Todos esses fatores associados a outros, como exposição do paciente a uma flora intra-hospitalar, tubagem traqueal prolongada, sobrecarga volêmica iatrogênica etc., fazem dos pulmões sede frequente de complicações que devem ser 
diagnosticadas precocemente e, fundamentalmente, prevenidas (5).

Dentre as complicações pulmonares pós-operatórias, a mais frequente é a atelectasia, que compromete um segmento, um lobo ou todo um pulmão (6). Chamase atenção para o fato de que, após cinco minutos de indução anestésica, pode-se observar, por tomografia computadorizada, a formação de atelectasia nas porções dependentes dos pulmões. 0 fluxo mucociliar também fica prejudicado por dois a seis dias após o ato anestésico, sendo que uma das complicações mais frequentes relacionadas à anestesia geral é o acúmulo de secreções nos pulmões, levando à formação de áreas de atelectasias e criando condições propícias ao desenvolvimento de infecções respiratórias (7).

Nesse contexto, a fisioterapia respiratória desempenha uma função relevante nas intervenções cirúrgicas, atuando na remoção de secreções pulmonares e na reexpansão pulmonar, utilizando como tratamento a pressão positiva expiratória (EPAP).

A EPAP é uma técnica eficaz em respiração espontânea, usada desde 1930, a qual visa aumentar a capacidade residual funcional (CRF), promover recrutamento alveolar, redistribuir a água extravascular, aumentar o volume e a pressão alveolar, mobilizar as secreções com o intuito de melhorar a oxigenação arterial, prevenir o colapso alveolar ou reverter quadros atelectásicos (8). Trata-se de uma terapêutica simples, de baixo custo e com enorme aplicação em patologias respiratórias, baseada na utilização de níveis de pressão positiva expiratória final (PEEP) $(9,10)$.

Assim, os objetivos do estudo descrito neste artigo foram propor um novo modelo de dispositivo EPAP subaquático e testar sua aplicabilidade em reverter atelectasias e complicações pós-operatórias em pacientes submetidos à cirurgia de RM.

\section{Materiais e métodos}

A amostra foi selecionada de forma não probabilística e intencional. Recrutaram-se todos os pacientes internados na Unidade Coronariana do Instituto de Cardiologia de Santa Catarina, na cidade de São José, com idade entre 55 e 65 anos e submetidos à cirurgia de RM de setembro a dezembro de 2006. Os critérios de inclusão foram: pacientes pós-desmame de ventilação mecânica, lúcidos, orientados e contactuantes, com sistemas cognitivos preservados e sem deformidades físicas orofaciais. Foram excluídos os pacientes obesos, dependentes de mais de 3 litros de oxigênio, com fratura torácica, sequelas neurológicas, complicações pós-cirúrgicas, com outras intervenções cirúrgicas adjuntas, ou submetidos à ventilação mecânica.

O novo modelo de dispositivo EPAP subaquático, como ilustra a Figura 1, foi construído em acrílico com um tubo pré-fabricado e uma mangueira com bocal de equipamento hospitalar (de um incentivador de fluxo chamado Respiron ${ }^{\circledR}$ ). 0 tubo de acrílico possui $230 \mathrm{~mm}$ de altura, $60 \mathrm{~mm}$ de diâmetro e $5 \mathrm{~mm}$ de espessura. A mangueira é sanfonada, com $325 \mathrm{~mm}$ de comprimento, $15 \mathrm{~mm}$ de diâmetro e um bocal na extremidade superior (Figuras 1A e 1B). Outras especificações do dispositivo: orifícios na tampa para escape de ar e para evitar pressão adicional (Figura 1C); orifício de encaixe da mangueira (Figura 1D); trava para evitar que a mangueira fosse retirada do fundo do tubo durante $o$ uso (Figura 1E); régua com números em tampografia para o nível de água (Figura 1F); rosca para facilitar a troca de água (Figura 1G); ranhuras para melhorar a preensão (Figura $1 \mathrm{H}$ ).

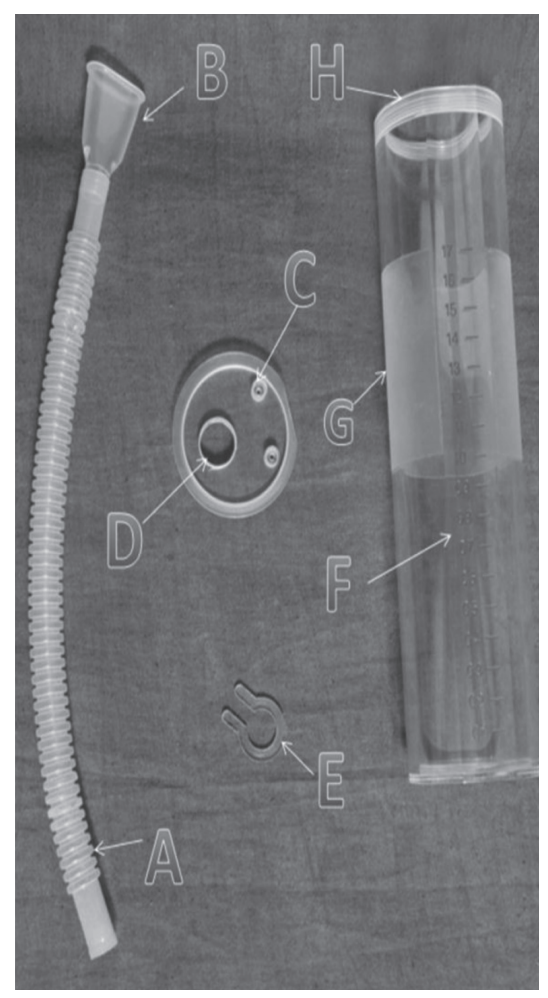

Figura 1 - Protótipo do dispositivo EPAP desenvolvido

Legenda: $A=$ mangueira; $B=$ bocal; $C=$ orifícios para escape de ar; $D=$ orifício de passagem da mangueira; $E=$ trava da mangueira; $F=$ régua numérica; $G=$ ranhuras; $H=$ rosca de encaixe da tampa.

Fonte: Dados da pesquisa. 
0 programa de tratamento proposto foi aprovado pelo Comitê de Ética do Instituto de Cardiologia e pelo Comitê de Ética da Pontifícia Universidade Católica do Paraná (PUCPR) vinculado ao Conselho Nacional de Ética em Pesquisa (Conep), sob protocolo n. 881. Os pacientes assinaram o Termo de Consentimento Livre e Esclarecido no pós-operatório logo após serem desmamados da ventilação mecânica, iniciando-se, então, a coleta de dados.

0 tratamento constou de quatro sessões com duração de 60 minutos em dois dias consecutivos, sendo duas sessões diárias: a primeira no início da manhã, e a segunda, no início da tarde. Após pesquisa do prontuário, análise das radiografias e preenchimento da ficha de avaliação, foi realizada a primeira sessão, observando-se o seguinte procedimento:

a) verificação dos sinais vitais: frequência cardíaca (FC), frequência respiratória (FR), saturação de oxigênio $\left(\mathrm{SpO}_{2}\right)$, pressão arterial (PA) e ausculta pulmonar;

b) durante a aplicação da "espirometria", usou-se clipe nasal e espirômetro portátil Micro $\mathrm{GP}^{\circledR}, \mathrm{e}$ solicitou-se que o paciente realizasse três expirações máximas, sendo considerado o maior valor das três. $\mathrm{A} \mathrm{SpO}_{2}$, a FC e a FR foram medidas no monitor da UTI. A posição adotada para o paciente, tanto para verificação dos parâmetros iniciais quanto para a aplicação da técnica EPAP, foi a fowler (sentado de $45^{\circ}$ a $60^{\circ}$, com travesseiro sob os joelhos semifletidos);

c) para a aplicação do protótipo "EPAP Subaquático", o paciente realizou uma inspiração máxima, seguida de uma breve apneia e, posteriormente, uma expiração prolongada sob o tubo imerso em $15 \mathrm{cmH}_{2} \mathrm{O}$, expirando completamente $\mathrm{o}$ ar, a coluna-d'água representa a pressão a ser fornecida aos pulmões. Efetuaram-se 20 repetições, com intervalos aproximados de 60 segundos, ou conforme as limitações do paciente. A respiração diafragmática foi estimulada durante todo o tratamento. E, no decorrer do procedimento, quando o paciente apresentou tosse produtiva e eficaz, analisou-se a quantidade e as características da secreção expectorada (11);

d) imediatamente após a sessão, os sinais vitais foram outra vez verificados;

e) utilizou-se a análise espirométrica na primeira e na quarta sessões, após 15 minutos de descanso; f) sendo a radiografia um procedimento diário de rotina em UTI, comparou-se a imagem radiográfica anterior à primeira e após a última sessão terapêutica aplicada; laudo fornecido pelo médico especialista.

O teste estatístico escolhido foi do tipo não paramétrico em razão da distribuição anormal dos dados, consequência do tamanho reduzido da amostra. Para amostras emparelhadas, realizou-se o teste de Wilcoxon. Na comparação entre sessões iniciais e finais, usou-se o teste para as comparações múltiplas de Friedman. Ainda foi utilizado, para análise da quantidade de secreções e ausculta pulmonar, o teste dos Sinais. Na aplicação dos testes, empregou-se o programa SPSS (Statistical Package for Social Science), versão 11.01, com nível de significância de $5 \%(\mathrm{p}<0,05)$.

\section{Resultados}

No período do estudo, foram avaliados 17 indivíduos. Desses, seis pacientes apresentavam as características necessárias para participar da pesquisa, conforme descrito no Quadro 1.

Porém, onze participantes foram excluídos da amostra e não puderam ser considerados para o estudo pelos seguintes motivos: complicações pós-cirúrgicas (vômitos, náuseas e dor nas incisões cirúrgicas) (sete pacientes); falta da assinatura no termo

Quadro 1- Características dos participantes da amostra

\begin{tabular}{lc}
\hline Características & Geral \\
\hline Participantes (n) & 6 \\
Sexo (Masc/Fem) & $3 / 3$ \\
Idade (anos) & $59,83(55-64)$ \\
Peso (Kg) & $66,5(51-72)$ \\
Estatura (cm) & $160(152-165)$ \\
Drenos (Mediastino/Torácico D/ & $6 / 2 / 4$ \\
Torácico E) & \\
Circulação Extracorpórea (CEC) & $3 / 3$ \\
(sim/não) & \\
Tabagista (sim/não/ex-tabagista) & $1 / 1 / 4$ \\
\hline
\end{tabular}

Fonte: Dados da pesquisa.

Nota: Os dados estão expressos como médias (valor mínimo - valor máximo). 
de consentimento por se negarem a participar do estudo (dois pacientes); e submissão a outras técnicas fisioterapêuticas durante o estudo (dois pacientes).

Os valores espirométricos medidos (CVF - capacidade vital forçada, $\mathrm{VEF}_{1}$ - volume expiratório forçado no primeiro segundo, $\mathrm{VEF}_{1} / \mathrm{CVF}$ - relação volume expiratório forçado no primeiro segundo e capacidade vital forçada, peak flow (PEF) ou fluxo expiratório máximo), comparando-se a primeira sessão e após a quarta sessão, não foram significativos $(\mathrm{p}<0,05)$. Os prováveis fatores que levaram a esse resultado incluem: tempo reduzido de permanência dos pacientes na UTI (apenas dois dias) e limitação ao fluxo expiratório em virtude da dor. Ademais, os pacientes apresentaram dificuldade em realizar inspirações profundas por fatores psicológicos, como o medo de romper a sutura cirúrgica, deslocar o dreno, além da dor para manter-se sentado e na incisão dos drenos.

Os sinais vitais, avaliados pelo estudo (FC, FR e PA), apresentaram mudanças mínimas quando se compararam os valores obtidos antes e após a aplicação do Protótipo EPAP Subaquático. 0 fato de o protótipo EPAP subaquático manter os sinais vitais sem alterações significativas indica que sua aplicação não produz modificações sobre a hemodinâmica do paciente, sendo segura sua aplicação.

Em relação à $\mathrm{SpO}_{2}$, os valores observados nas sessões iniciais apresentam um valor de p significativo ( $\mathrm{p}=0,007$ ), como ilustra o Quadro 2 .

Da mesma forma, as sessões finais, quando comparadas às iniciais, também apresentaram diferenças significativas ( $p=0,03)$, de acordo com o teste de Friedman.
Pode-se notar que a média para a sessão inicial e a sessão final foram, respectivamente, 91,3 $\pm 1,4$ e 98,0 \pm 0,6. Houve aumento significativo $(\mathrm{p}<0,05)$, verificado pelo teste de Wilcoxon $(p=0,03)$ como mostra a Figura 2 .

A análise das radiografias foi realizada de forma subjetiva e comparativa, a radiografia inicial, antes da intervenção fisioterapêutica com a aplicação do protótipo EPAP subaquático, com a radiografia final, após a aplicação da técnica, cujas imagens encontram-se ilustradas na Figura 3.

Ao comparar as radiografias, considerou-se a relação de volume pulmonar e hipotransparência, pois as técnicas radiológicas utilizadas nem sempre foram as mesmas. Entretanto, constata-se melhora por meio das radiografias finais em relação às iniciais, com áreas mais ventiladas e perfundidas e diafragma em posição mais próxima à normalidade.

Em relação à ausculta pulmonar, segundo a análise estatística do Teste dos Sinais, a comparação entre a ausculta inicial, antes do início da aplicação do protótipo EPAP subaquático e seu término, o valor estatístico de $\mathrm{p}=0,0156$ denotou significância. Observa-se melhora na ausculta do murmúrio vesicular e diminuição dos ruídos adventícios após a aplicação do dispositivo desenvolvido.

Evidencia-se a relação existente entre as áreas pouco ventiladas nas radiografias, que tendem a ser áreas de atelectasias, a diminuição do murmúrio vesicular bilateral auscultado em todos os pacientes da pesquisa no momento da avaliação e a presença de estertores crepitantes, com a quantidade de secreção expectorada durante as primeiras sessões.

Quadro 2- Estatística descritiva dos valores de $\mathrm{SpO}_{2}$ nas sessões iniciais

\begin{tabular}{|c|c|c|c|c|c|}
\hline & & Sessão 1 & Sessão 2 & Sessão 3 & Sessão 4 \\
\hline N & & 6 & 6 & 6 & 6 \\
\hline $\bar{x}$ & & 91,3 & 92,3 & 92,7 & 94,7 \\
\hline$S$ & & 1,4 & 1,0 & 2,4 & 1,2 \\
\hline Mínimo & & 90,0 & 91,0 & 90,0 & 93,0 \\
\hline Máximo & & 93,0 & 94,0 & 96,0 & 96,0 \\
\hline \multirow[t]{3}{*}{ Percentis } & 25 & 90,0 & 91,8 & 90,0 & 93,8 \\
\hline & 50 & 91,0 & 92,0 & 93,0 & 94,5 \\
\hline & 75 & 93,0 & 93,3 & 94,5 & 96,0 \\
\hline
\end{tabular}

Fonte: Dados da pesquisa.

Nota: Os dados estão expressos como médias (valor mínimo - valor máximo). 


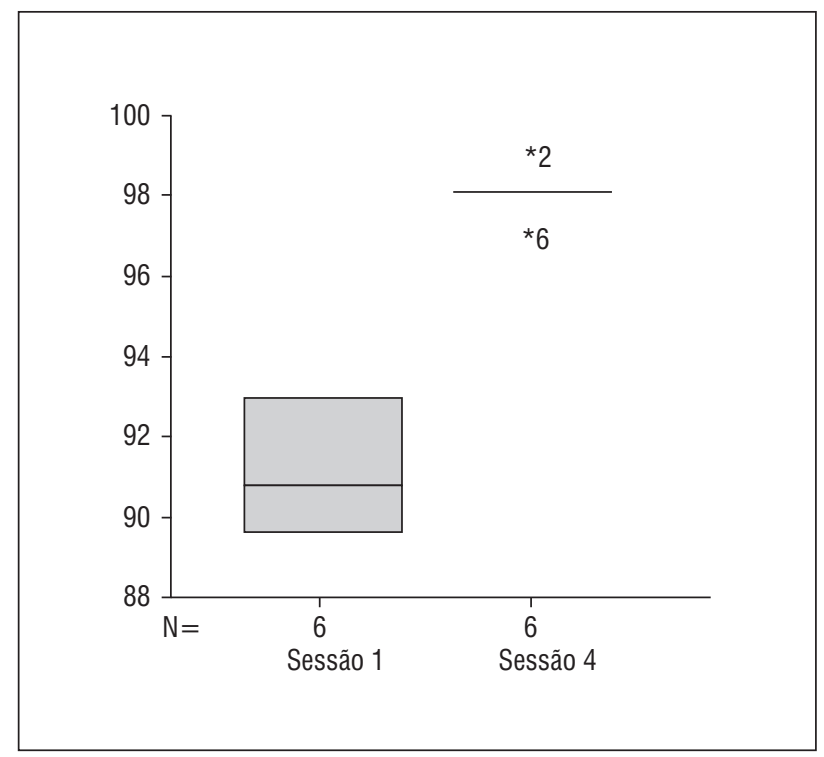

Figura 2 - Resultado dos valores de $\mathrm{SpO}_{2}$ sessão 1 (inicial) e sessão 4 (final)

Fonte: Dados da pesquisa.

0 valor estatístico de $\mathrm{p}=0,0156$ indica significância nos resultados encontrados em relação à expectoração da secreção pulmonar. Na primeira sessão, observou-se grande expectoração, mas na quarta, os pacientes já estavam sem expectoração.

\section{Discussão}

No pós-operatório de RM, ocorrem importantes alterações dos parâmetros da função pulmonar, cujas reversões são lentas, requerendo o uso de técnicas ou recursos fisioterápicos associados à aplicação de pressão positiva nas vias aéreas com a finalidade de reduzir complicações decorrentes da cirurgia (12). No entanto, constatou-se que mesmo com a aplicação de pressão positiva nas vias aéreas, os valores espirométricos não são totalmente restabelecidos até a alta hospitalar, confirmando os valores encontrados no segundo e terceiro dias de pós-operatório em que foi realizada a medida e os valores, que se encontram bem abaixo da normalidade (12).

Alterações na função pulmonar ocorrem em todos os pacientes, horas depois do processo cirúrgico, e a redução dos volumes pulmonares pode ser observada, principalmente, do pré para o primeiro dia de pós-operatório, havendo aumento, mas não retorno aos valores pré-operatórios, no sexto dia pós-operatório (13). Acredita-se que se a espirometria final fosse realizada com um intervalo mais prolongado - após o sexto dia de pós-operatório, por exemplo - os resultados seriam melhores.

Os parâmetros espirométricos finais comparados aos iniciais não apresentaram diferenças estatisticamente significativas. A queda do Peak Flow tem importância clínica, pois mede a eficácia da função pulmonar e revela o fechamento precoce e a obstrução das pequenas vias aéreas que predispõem às microatelectasias, também, reflete a capacidade de tossir, debilita a tosse e o transporte de secreções. Há indícios de que a limitação em relação à dor e à respiração superficial, evidente em todos os pacientes de pós-operatório de cirurgia cardíaca, dificulta a realização do exame espirométrico de maneira precisa, o que justifica o fato de não haver alterações de valores espirométricos. Ou seja, o protótipo EPAP subaquático não se mostrou eficaz em melhorar a função pulmonar nos pacientes de RM.

Não se verificaram neste trabalho os valores espirométricos pré-operatórios. Abordou-se o paciente no segundo e terceiro dias após a cirurgia, em um intervalo de tempo muito reduzido, sendo um dos fatores importantes por não produzir diferenças significativas entre as variáveis.

Em um estudo relatado por Spigolon et al. (14), averiguou-se o período dos efeitos da terapia EPAP sobre as variáveis saturação de oxigênio, frequência cardíaca e respiratória, em nove pacientes com diagnóstico de Infarto Agudo do Miocárdio, em até 72 horas. Em tal estudo, com PEEP de até $10 \mathrm{cmH}_{2} \mathrm{O}$, tanto a FC quanto a FR antes e após a utilização do EPAP não apresentaram diferença clínica, mas promoveram elevação dos níveis de saturação de oxigênio (14).

Os sinais vitais mantiveram-se dentro dos padrões de normalidade recomendados pela literatura, mesmo após a aplicação do EPAP subaquático, portanto, o dispositivo não provoca alterações significativas em frequência cardíaca, frequência respiratória e pressão arterial, o que é clinicamente importante, pois qualquer alteração hemodinâmica pode ser prejudicial ao paciente.

Quando são analisados os resultados de saturação de oxigênio, verifica-se que todas as medidas apresentaram diferenças significativas $(\mathrm{p}<0,05)$. Sob o aspecto clínico, isso ressalta a melhora do paciente em relação ao cansaço físico e à dor, pois após a aplicação do EPAP subaquático, além de aumentar a intensidade da respiração, tornando-a menos 


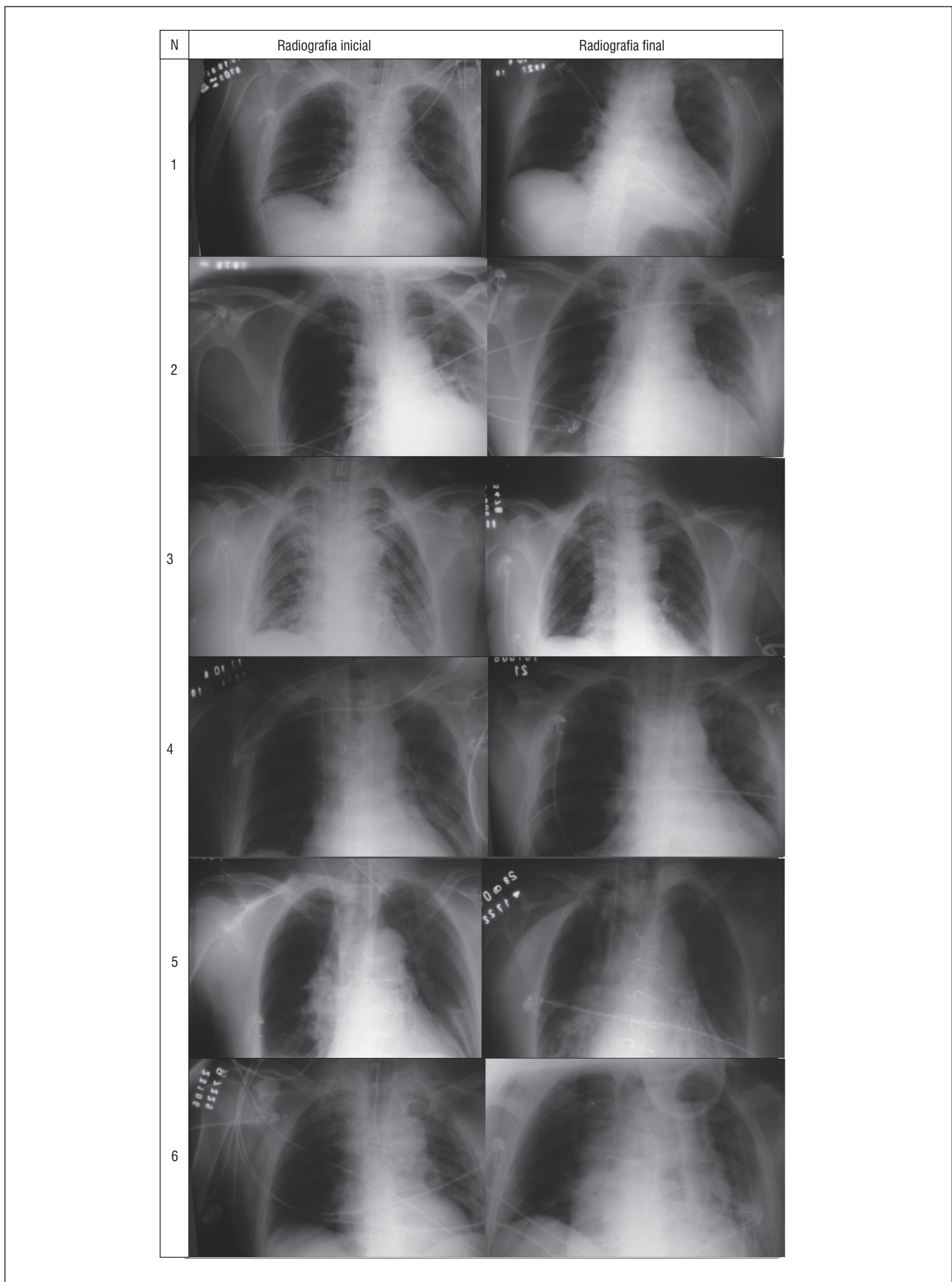

Figura 3 - Radiografias iniciais comparadas às radiografias finais dos seis pacientes avaliados Fonte: Dados da pesquisa. 
superficial, eleva-se o volume de ar inspirado, melhorando a saturação de oxigênio e a mobilidade torácica, que diminui a dor nas incisões cirúrgicas.

Sendo assim, o protótipo mostrou-se muito eficaz, reduzindo áreas de shunt e melhorando o recrutamento alveolar. A melhora na oxigenação também é comprovada quando se compara seus resultados significativos com a melhora de áreas de atelectasia das radiografias e remoção das secreções pulmonares que dificultam as trocas gasosas, indicando que o uso de pressão positiva expiratória final melhora a oxigenação pulmonar.

Os achados do aumento da saturação de oxigênio ratificam-se em estudos o como de Miranda e Santos (15), no qual foram analisados os efeitos do EPAP no pós-operatório de 18 pacientes submetidos à RM, a partir de resultados da gasometria arterial e oximetria de pulso. 0 grupo de pacientes que realizava o EPAP subaquático obteve resultados significativos no aumento da Pressão Parcial de Oxigênio $\left(\mathrm{PaO}_{2}\right)$ e na diminuição da Pressão Parcial de Dióxido de Carbono $\left(\mathrm{PaCO}_{2}\right)$, melhorando, com isso, o nível de oxigenação, quando comparado com o grupo que realizou apenas fisioterapia convencional, sem aplicação do EPAP (15).

O uso terapêutico da PEEP vem sendo amplamente difundido, visando ao aumento da oxigenação arterial, à redução do shunt, à melhora da Capacidade Residual Funcional e, consequentemente, ao recrutamento alveolar, melhorando a $\mathrm{SpO}_{2}(8)$.

0 EPAP subaquático contribui na melhora da ventilação em áreas que não estavam sendo ventiladas, principalmente pela pressão positiva, e sua relação com os canais colaterais evidenciados nos pulmões, os quais são encontrados em nível alveolar (chamados poros de Kohn), entre brônquios e alvéolos (canais de Lambert) e entre bronquíolos (canais interbronquiolares de Martin), promovendo maior eliminação das secreções (16). Em trabalhos utilizando CPAP no tratamento de atelectasias, relata-se que os seguintes fatores contribuem com seus efeitos benéficos: recrutamento de alvéolos colapsados através de um aumento da CRF; diminuição do trabalho respiratório em razão do aumento da complacência ou abolição da auto-PEEP; melhor distribuição da ventilação através dos canais colaterais; e aumento da eficácia de remoção de secreções (17).

Neste estudo, o protótipo EPAP subaquático melhorou a ventilação pulmonar revertendo áreas de atelectasia previamente detectadas nas radiografias iniciais, quando comparadas às finais, ou seja, é eficaz em melhorar áreas hipoventiladas e atelectásicas. Também foi efetivo na expectoração de secreção em relação à ventilação colateral que desloca as secreções quando os pulmões são submetidos a pressões positivas; portanto, pode ser utilizado em pós-operatório quando o objetivo é a higiene brônquica.

Constata-se, ainda, além dos benefícios já citados com a aplicação da técnica de EPAP subaquático, que a montagem do aparelho projetado tornou-se fácil com o uso do sistema de rosca. Além disso, com o calibre da mangueira próximo ao tamanho da traqueia, não se cria resistência adicional; funciona como um prolongamento da traqueia durante a expiração. Quanto menor o calibre da mangueira, maior seria a pressão fornecida ao sistema; dessa forma, consegue-se impor pela coluna-d'água apenas a pressão que se deseja.

0 protótipo desenvolvido proporcionou melhora efetiva da ausculta pulmonar, evidenciando melhora no murmúrio vesicular e ruídos adventícios após uso do dispositivo. Ademais, foi efetivo em diminuir as queixas dos pacientes, uma vez comparadas a primeira e a última sessão, principalmente pelo trabalho de reexpansão pulmonar que, consequentemente, melhora a postura no leito, promove alongamento da musculatura respiratória e facilita a inspiração.

\section{Conclusão}

A partir da aplicação clínica do dispositivo desenvolvido, pode-se concluir que o dispositivo não provoca alterações significativas nas frequências cardíaca e respiratória e na pressão arterial, o que é clinicamente relevante, pois qualquer alteração hemodinâmica pode ser prejudicial ao paciente.

Em relação à saturação de oxigênio, o protótipo mostrou-se eficaz, reduzindo áreas de shunt e melhorando o recrutamento alveolar, demonstrando, assim, que o uso de pressão positiva expiratória final melhora a oxigenação pulmonar. Além disso, ele melhora a ventilação pulmonar revertendo áreas hipoventiladas e atelectásicas.

O dispositivo foi efetivo na expectoração de secreção em relação à ventilação colateral que desloca as secreções quando os pulmões são submetidos a pressões positivas, indicando, desse modo, que 
pode ser utilizado em pós-operatório quando se objetiva higiene brônquica. Por meio da ausculta pulmonar, evidenciaram-se melhoras no murmúrio vesicular e ruídos adventícios com o uso do protótipo testado.

Não houve alterações nos parâmetros espirométricos, ou seja, o protótipo EPAP subaquático não se mostrou eficaz em melhorar a função pulmonar nos pacientes de RM, porém diminuiu as queixas dos pacientes ao longo do tratamento, indicando melhora da postura no leito, alongamento da musculatura respiratória e facilitação da inspiração. Por fim, o aparelho é de fácil montagem, manuseio e higienização, além de permitir reutilização.

\section{Referências}

1. Sociedade Paulista de Pneumologia e Tisiologia. Pneumologia - atualização e reciclagem. Vol. 2. São Paulo: Atheneu; 1998.

2. Ignaszewski S, Lear SA. Cardiac Rehabilitation programs. Can J Cardiol. 1999;15(SupplG):110G-3G. PMID: 10692670

3. Pasquina P, Tramér MR, Walder B. Prophylactic respiratory physiotherapy after cardiac surgery: systematic review. BMJ. 2003;327(7428):1379. doi:10.1136/ bmj.327.7428.1379.

4. Netto CA. Clínica Cirúrgica. 4. ed. Vol. 3. São Paulo: Sarvier; 1998.

5. Jorge I Filho, Andrade JI, Zillioto A Júnior. Cirurgia Geral. Pré e pós-operatório. São Paulo: Atheneu; 1996.

6. Lima MRO, Freire ALG, Andrade LB, Leopoldino GS. Comparação dos níveis de pressão positiva contínua nas vias aérea através de dois sistemas. J. Pediatr. 2004;80(5):401-6. doi:10.2223/JPED.1225.

7. Paschoal IA, Pereira MC. Controle Clínico do paciente cirúrgico para procedimentos não cardíacos - abordagem pré-operatória do paciente pneumopata: riscos e orientações. Rev Soc Cardiol Estado de São Paulo. 2001;10(3):157-65.

8. Publio AZ, Tuzi DA, Passoni HC, Rocha SM. Estudo da pressão positiva expiratória e sua eficácia na atelectasia: uma revisão de literatura. Fisioter Mov. 2004;17(4):43-50.
9. Regenga MM. Fisiologia em cardiologia: da unidade de terapia intensiva à reabilitação. São Paulo: Roccan; 2000.

10. Azeredo CAC. Fisioterapia respiratória moderna. São Paulo: Atheneu; 1993.

11. Costa D. Fisioterapia respiratória básica. São Paulo: Atheneu; 1999.

12. Silva LCC. Condutas em Pneumologia. Vol. 1. Rio de Janeiro: Revinter; 2001.

13. Leguisamo CP, Kalil RAK, Furlani AP. A efetividade de uma proposta fisioterapêutica pré-operatória para cirurgia de revascularização do miocárdio. RBCCV. 2005;20(2):134-41.

14. Spigolon N, Ambrozin A, Carvalho G, Caminha P. Aplicação do EPAP em pacientes com Infarto Agudo do Miocárdio. Rev Bras Fisioter. 2004;Supl 185.

15. Miranda A, Santos M. Análise dos efeitos da pressão positiva expiratória nas vias aéreas no pós-operatório de pacientes submetidos a revascularização do miocárdio. Anais do XII Simpósio Internacional de Fisioterapia Respiratória. Ouro Preto - MG; 2004.

16. Fink JB. Positive pressure techniques for airway clearance. Resp Care. 2002;47:789-96.

17. Scanlan CL, Wilkins RL, Stoller JK. Fundamentos da terapia respiratória de Egan. 7. ed. São Paulo: Manole; 2000
Recebido: 08/04/2012

Received: 04/08/2012

Aprovado: 03/11/2012

Approved: 11/03/2012 